\title{
Minimal definable graphs of definable chromatic number at least three
}

\author{
Raphaël Carroy ${ }^{1}$, Benjamin D. Miller ${ }^{2}$, David Schrittesser ${ }^{3}$ and Zoltán Vidnyánszky ${ }^{4}$ \\ ${ }^{1}$ Raphaël Carroy, Dipartimento di Matematica "Giuseppe Peano", Università degli studi di Torino Palazzo Campana, Via Carlo \\ Alberto 10, 10123 Torino, Italy, E-mail: raphael.carroy@univie.ac.at; http://www.logique.jussieu.fr/ carroy/indexeng.html. \\ ${ }^{2}$ University of Vienna, Department of Mathematics, Oskar Morgenstern Platz 1, Wien 1090, \\ Austria, E-mail: benjamin.miller@univie.ac.at; https://homepage.univie.ac.at/benjamin.miller/. \\ ${ }^{3}$ University of Vienna, Department of Mathematics, Oskar Morgenstern Platz 1, Wien 1090, \\ Austria, E-mail: david.schrittesser@univie.ac.at; http://homepage.univie.ac.at/david.schrittesser/. \\ ${ }^{4}$ University of Vienna, Department of Mathematics, Oskar Morgenstern Platz 1, Wien 1090, Austria; and California Institute of \\ Technology, Department of Mathematics, Pasadena, CA 91125, E-mail: zoltan.vidnyanszky@univie.ac.at; \\ http://www.logic.univie.ac.at/ vidnyanszz77/.
}

Received: 26 November 2019; Revised: 14 September 2020; Accepted: 5 October 2020

2020 Mathematics Subject Classification: Primary - 03E15, 28A05

Keywords and phrases: Borel bipartite, Borel coloring

\begin{abstract}
We show that there is a Borel graph on a standard Borel space of Borel chromatic number three that admits a Borel homomorphism to every analytic graph on a standard Borel space of Borel chromatic number at least three. Moreover, we characterize the Borel graphs on standard Borel spaces of vertex-degree at most two with this property and show that the analogous result for digraphs fails.
\end{abstract}

\section{Contents}

1 Introduction

2 Preliminaries and basic facts

3 A basis under continuous reducibility $\quad 7$

4 Large gaps $\quad 10$

5 An antibasis result for digraphs $\quad 13$

6 Open problems $\quad 15$

$\begin{array}{lr}\text { References } & 16\end{array}$

\section{Introduction}

The investigation of definable chromatic numbers is a blooming field of research with numerous applications, as can be found in [2, 6, 7, 8, 15, 16, 17, 18, 23]. The survey [13] contains many of the latest results.

Recall that a digraph on a set $X$ is an irreflexive set $G \subseteq X^{2}$, and a graph on $X$ is a symmetric digraph on $X$. A $\kappa$-coloring of a digraph $G$ on $X$ is a map $c: X \rightarrow \kappa$ such that $(x, y) \in G \Longrightarrow c(x) \neq c(y)$ for 
all $x, y \in X$. We will be interested in digraphs on spaces $X$ that are endowed with a Borel structure. In this case, one may consider the Borel chromatic number of $G$, or $\chi_{B}(G)$, defined as the least cardinal $\kappa$ that admits a standard Borel structure with respect to which there is a Borel $\kappa$-coloring of $G$. (Note that a standard Borel structure exists on $\kappa$ iff $\kappa \in\left\{0,1,2, \ldots, \boldsymbol{\aleph}_{0}, 2^{\boldsymbol{N}_{0}}\right\}$, and for each such $\kappa$, it is unique up to Borel isomorphism.)

A homomorphism from a digraph $G$ on $X$ to a digraph $G^{\prime}$ on $X^{\prime}$ is a map $\phi: X \rightarrow X^{\prime}$ such that $(x, y) \in G \Longrightarrow(\phi(x), \phi(y)) \in G^{\prime}$ for all $x, y \in X$. When $G$ and $G^{\prime}$ are digraphs on standard Borel spaces, we write $G \leq_{B} G^{\prime}$ to indicate the existence of a Borel homomorphism from $G$ to $G^{\prime}$. Similarly, when $G$ and $G^{\prime}$ are digraphs on Polish spaces, we write $G \leq_{c} G^{\prime}$ to indicate the existence of a continuous homomorphism from $G$ to $G^{\prime}$. It is easy to see that $G \leq_{B} G^{\prime} \Longrightarrow \chi_{B}(G) \leq \chi_{B}\left(G^{\prime}\right)$. The complete graph on $\kappa$ is given by $K_{\kappa}=\left\{(\alpha, \beta) \in \kappa^{2} \mid \alpha \neq \beta\right\}$. It is also easy to see that if $\kappa$ is endowed with a standard Borel structure, then $\chi_{B}(G) \leq \kappa \Longleftrightarrow G \leq_{B} K_{K}$.

The systematic investigation of Borel chromatic numbers was initiated by Kechris, Solecki and Todorčevic [14]. One of their primary successes was the isolation of a Borel graph $\mathbb{G}_{0}$ on $2^{\mathbb{N}}$ of uncountable Borel chromatic number that admits a continuous homomorphism to every analytic Borel graph on a Polish space of uncountable Borel chromatic number. This result lies at the heart of a vast number of seemingly unrelated theorems in descriptive set theory (see, e.g. [4, 5, 19, 21, 22]), often yielding shorter, more elegant proofs and substantial generalizations. Todorčevic and Vidnyánszky [25] recently ruled out the most straightforward analogs of the $\mathbb{G}_{0}$ dichotomy for graphs of Borel chromatic number at least $n$, where $4 \leq n \leq \boldsymbol{\aleph}_{0}$.

We will introduce a Borel graph $\mathbb{L}_{0}$ that plays a role analogous to $\mathbb{G}_{0}$ for graphs of Borel chromatic number at least three:

Theorem 1.1. Suppose that $G$ is an analytic graph on a Polish space. Then exactly one of the following holds:

(1) The graph $G$ has Borel chromatic number at most two.

(2) There is a continuous homomorphism from $\mathbb{L}_{0}$ to $G$.

It is easy to see that there is no analogous finite basis in the case of finite graphs, where the notions of Borel graph and Borel chromatic number coincide with their classical counterparts.

The graph $\mathbb{L}_{0}$ can be described using an inverse limit-like construction as follows: let $X_{0,0}$ be a twopoint set, let $L_{0,0}$ be the unique connected graph on $X_{0,0}$, and define $X_{0}=X_{0,0}$. Given $n \in \mathbb{N}$, a finite set $X_{0, n}$, and a tree $L_{0, n}$ on $X_{0, n}$ of vertex degree at most two, let $X_{0, n+1}$ be the disjoint union of two copies of $X_{0, n}$ with a set $X_{n+1}$ of cardinality $2 n+2$, fix a point $s_{n} \in X_{0, n}$ of $L_{0, n}$-vertex degree one, fix a tree $L_{n+1}$ on $X_{n+1}$ of vertex degree at most two, and let $L_{0, n+1}$ be the graph on $X_{0, n+1}$ whose restriction to each copy of $X_{0, n}$ is the corresponding copy of $L_{0, n}$, whose restriction to $X_{n+1}$ is $L_{n+1}$, and which connects the two copies of $s_{n}$ in $X_{0, n+1}$ to distinct points of $X_{n}$ of $L_{n}$-vertex degree one (see Figure 1). Let $\pi_{n+1}: X_{0, n+1} \backslash X_{n+1} \rightarrow X_{0, n}$ be the projection sending each point in one of the two copies of $X_{0, n}$ within $X_{0, n+1}$ to the corresponding point of $X_{0, n}$. Let $\mathbb{X}_{0}$ be the set of pairs of the form $(n, x)$, where $n \in \mathbb{N}$ and $x \in X_{n} \times \prod_{m>n} X_{0, m}$, such that $x(m)=\pi_{n+m+1}(x(m+1))$ for all $m \in \mathbb{N}$. Let $\mathbb{L}_{0}$ be the graph on $\mathbb{X}_{0}$ consisting of all pairs $\left((n, x),\left(n^{\prime}, x^{\prime}\right)\right) \in \mathbb{X}_{0} \times \mathbb{X}_{0}$ with the property that $\left(x(m), x^{\prime}(m)\right) \in L_{0, m}$ for all $m \geq \max \left(n, n^{\prime}\right)$. We will give a slightly different description of this graph in Section 2.

Our proof of Theorem 1.1 splits into two parts: we first establish the existence of continuum-many $\mathbb{L}_{0}$-like Borel digraphs that serve as a basis for the analytic digraphs on Polish spaces of Borel chromatic number at least three under continuous homomorphism, and then we show that the undirected version of any of these digraphs admits a continuous homomorphism to the undirected version of any other.

Suppose that $X$ is a set and $L$ is a graph on $X$ of vertex degree at most two. We say that a set $Y \subseteq X$ has large gaps if every $L$-component contains $L$-connected sets disjoint from $Y$ of arbitrarily large finite cardinality. When $X$ is a standard Borel space, we say that $L$ has the large gap property if there is a Borel set $B \subseteq X$ with large gaps that intersects every $L$-component. We say that $L$ has the large gap property modulo a two-colorable set if there is an $L$-invariant Borel set $M \subseteq X$ such that $L \uparrow(X \backslash M)$ has the 

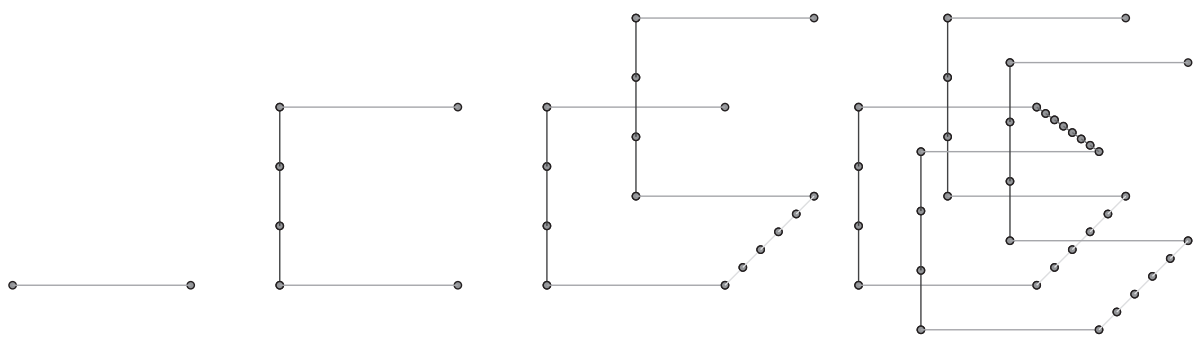

Figure 1. The first four stages of the construction of $\mathbb{L}_{0}$.

large gap property and $\chi_{B}(L \uparrow M) \leq 2$. We also characterize the family of Borel graphs $L$ on standard Borel spaces of vertex degree at most two satisfying the analog of Theorem 1.1 in which the existence of a continuous homomorphism from $\mathbb{L}_{0}$ to $G$ is replaced with the existence of a Borel homomorphism from $L$ to $G$ :

Theorem 1.2. Suppose that $X$ is a standard Borel space and $L$ is an acyclic Borel graph on $X$ of vertex degree at most two. Then the following are equivalent:

(1) There is a Borel homomorphism from $L$ to every Borel graph $G$ of Borel chromatic number at least three.

(2) The graph L has the large gap property modulo a two-colorable set.

An oriented graph on a set $X$ is an antisymmetric digraph on $X$. Whereas the oriented analog of $\mathbb{G}_{0}$ satisfies the analog of the Kechris-Solecki-Todorčevic dichotomy for analytic digraphs, we also show that there is no such analog of Theorem 1.1:

Theorem 1.3. Suppose that $G$ is an analytic digraph on a Polish space of Borel chromatic number at least three. Then there is a sequence $\left(L_{t}\right)_{t \in 2^{\mathbb{N}}}$ of Borel oriented graphs on Polish spaces of Borel chromatic number three that admit continuous homomorphisms to G but for which every analytic digraph on a standard Borel space that admits a Borel homomorphism to at least two distinct graphs of the form $L_{t}$ has Borel chromatic number at most two.

One can view $\mathbb{L}_{0}$ as being built via towers over a canonical acyclic graph $L$ on $2^{\mathbb{N}}$ of vertex degree at most two that is not the graph of a Borel function. In a future paper, we will establish a basis theorem for the analytic graphs on Polish spaces of Borel chromatic number at least three under the finer notion of injective continuous homomorphism. While the cardinality of the basis we will provide is necessarily (at least) that of the continuum, its elements are reminiscent of $\mathbb{L}_{0}$, in that they too can be viewed as being built via towers, albeit this time over three canonical graphs: the graph $L$ over which $\mathbb{L}_{0}$ is built, the graph of the odometer on $2^{\mathbb{N}}$ and the graph of the unilateral shift on increasing $\mathbb{N}$-sequences of natural numbers (for a summary of the results, see [3]).

In Section 2, we collect the most important definitions and facts used in our arguments. In Section 3 , we give the first half of the proof of Theorem 1.1. In Section 4, we give the second half and establish Theorem 1.2. In Section 5, we establish our anti-basis result. In Section 6, we discuss open problems.

\section{Preliminaries and basic facts}

We refer the reader to [12] for general background on descriptive set theory.

For each ordered pair $(x, y)$, set $(x, y)^{1}=(x, y)$ and $(x, y)^{-1}=(y, x)$. Define $B^{-1}=\left\{(x, y)^{-1}\right.$ | $(x, y) \in B\}$ for all sets $B \subseteq X^{2}$. Given a digraph $G$ on a set $X$ and $x, y \in X$, an (undirected) $G$-walk from $x$ to $y$ is a pair $p=\left(\left(x_{0}, \ldots, x_{\ell}\right), d_{p}\right)$ consisting of a finite sequence of vertices $\left(x_{0}, \ldots, x_{\ell}\right)$ with $x_{0}=x$ and $x_{\ell}=y$, and $d_{p} \in\{ \pm 1\}^{\ell}$ such that $\left(x_{i}, x_{i+1}\right)^{d_{p}(i)} \in G$ for all $i<\ell$. In the case that $p$ is a $G$-walk and $G$ is a graph, we will omit the second coordinate of $p$. 
For all $d \in\{ \pm 1\}^{<\mathbb{N}}$, we use $\Sigma(d)$ to denote $\sum_{i \in \operatorname{dom}(d)} d(i)$. We set dilength $(p)=\Sigma\left(d_{p}\right)$ and length $(p)=\ell$ for the directed length and (undirected) length of $p . \operatorname{Let}_{\operatorname{dist}_{G}}(x, y)$ be the minimal length of a $G$-walk from $x$ to $y$.

It is easy to verify the next claim.

Claim 2.1. Let $G$ be an acyclic ${ }^{1}$ oriented graph on the space $X$, and $x, y \in X$. Then for any two $G$-walks $p$ and $p^{\prime}$ from $x$ to $y$, we have $\operatorname{dilength}(p)=\operatorname{dilength}\left(p^{\prime}\right)$.

Thus, for an oriented acyclic graph $G$ on the space $X$ and $x, y \in X$, defining $\operatorname{didist}^{G}(x, y)$ to be the directed length of a walk from $x$ to $y$ makes sense. If it is clear from the context, we will omit the superscript from $\operatorname{dist}(\cdot, \cdot)$ and $\operatorname{didist}(\cdot, \cdot)$.

Note also that the parity of $\operatorname{dilength}(p)$ and length $(p)$ is the same. So, when referring to the parity of the length of a walk, we will always omit the word directed.

Define an equivalence relation $E_{G}$ on $X$ by letting $x E_{G} y$ iff there exists a $G$-walk from $x$ to $y$. The $E_{G}$ equivalence classes will be called the connected components or components of $G$. For standard definitions and facts from the theory of equivalence relations (e.g. smoothness, saturation and countability), see [10]. As usual, a set $S \subseteq X$ will be called $G$-invariant if it is $E_{G}$-invariant.

The restriction of $G\left(E_{G}\right)$ to $B$, in notation $G \uparrow B\left(E_{G} \uparrow B\right)$, is the digraph $G \cap B^{2}$ (the equivalence relation $E_{G} \cap B^{2}$ ) on $B$. A set $B \subseteq X$ is called $G$-independent if $B^{2} \cap G=\emptyset$.

Definition of $\mathbb{L}_{0}$-type graphs. Now we outline a general scheme for constructing Borel graphs; the graph $\mathbb{L}_{0}$ will be a particular example of such a construction. First, we define finitary approximations to our graphs, parametrized by a sequence $c \in \mathbb{N}^{\mathbb{N}}$. For all $n \in \mathbb{N}$, let $L_{n}$ denote the graph on $\{(0), \ldots,(n)\}$ with respect to which $(i)$ and $(j)$ are neighbors if and only if $|i-j|=1$ (here, the symbol $(s)$ stands for the sequence containing a single element $s$ ). For the rest of the paper, we fix a sequence $\left(s_{n}\right)_{n \in \mathbb{N}}$ given by $s_{0}=(c(0))$ and $s_{n}=(0)^{n}-(1)$, for $n>0$. Define graphs $L_{c, n}$ on $X_{c, n}=\bigcup_{m \leq n}\{0, \ldots, c(m)\} \times 2^{n-m}$ by setting $L_{c, 0}=L_{c(0)}$ and letting $L_{c, n+1}$ be the acyclic connected graph, containing $\left\{\left(v_{i}-(j)\right)_{i<2} \mid j<2\right.$ and $\left.\left(v_{i}\right)_{i<2} \in L_{c, n}\right\}$ and $L_{c(n+1)}$, in which $\left(s_{n}, 0\right)$ is a neighbor of $(0)$ and $(c(n+1))$ is a neighbor of $\left(s_{n}, 1\right)$.

Now set $\mathbb{X}_{c}=\left\{(n, k, r) \in \mathbb{N} \times \mathbb{N} \times 2^{\mathbb{N}} \mid k \leq c(n)\right\}$, define $\pi_{c, n}: \mathbb{X}_{c} \cap\left(\{0, \ldots, n\} \times \mathbb{N} \times 2^{\mathbb{N}}\right) \rightarrow X_{c, n}$ by $\pi_{c, n}(m, k, r)=(k)-r \uparrow(n-m)$ for all $n \in \mathbb{N}$, and let $\mathbb{L}_{c}$ be the graph on $\mathbb{X}_{c}$ consisting of all pairs of the form $\left(\left(n_{i}, k_{i}, r_{i}\right)\right)_{i<2}$ such that $\left(\pi_{c, n}\left(n_{i}, k_{i}, r_{i}\right)\right)_{i<2} \in L_{c, n}$ holds $\forall n \geq \max \left(n_{0}, n_{1}\right)$.

Recall that, in the introduction, we described the graph $\mathbb{L}_{0}=\mathbb{L}_{c}$ with $c(0)=1$ and $c(n)=2 n-1$ for $n>0$.

Definition of $\mathbb{L}_{0}$-type oriented graphs. We modify the preceding construction slightly, considering oriented finitary approximations, which yield oriented Borel graphs as limits.

An extra parameter is necessary to encode the orientation of the graphs. For all $n \in \mathbb{N}$ and $d \in\{-1,1\}^{k}$ with $k>n$, let $L_{n}^{d}$ denote the oriented graph on $\{(0), \ldots,(n)\}$ containing $((i),(j))^{d(\max \{i, j\})}$ whenever $|i-j|=1$.

Let $n \in\left\{0,1, \ldots, \boldsymbol{\aleph}_{0}\right\}$. To ease notation, we will call a pair $b=(c, d)$ an odd n-pair if $c: 1+n \rightarrow$ $2 \mathbb{N}+1, d: 1+n \rightarrow\{-1,1\}^{<\mathbb{N}}$ and $|d(k)|=c(k)+2$ for all $k \leq n$.

Given an odd $\aleph_{0}$-pair $b=(c, d)$, define graphs $L_{b, n}$ on $X_{c, n}$ by setting $L_{b, 0}=L_{c(0)}^{d(0)}$ and letting $L_{b, n+1}$ be the acyclic connected oriented graph, containing $\left\{\left(v_{i}-(j)\right)_{i<2} \mid j<2\right.$ and $\left.\left(v_{i}\right)_{i<2} \in L_{b, n}\right\}$ and $L_{c(n+1)}^{d(n+1)}$, in which

$$
\left.\left(\left(s_{n}, 0\right),(0)\right)\right)^{d(n+1)(0)} \text { and }\left((c(n+1)),\left(s_{n}, 1\right)\right)^{d(n+1)(c(n+1)+1)}
$$

are edges. Finally, let $\mathbb{L}_{b}$ be the graph on $\mathbb{X}_{c}$ consisting of all pairs of the form $\left(\left(n_{i}, k_{i}, r_{i}\right)\right)_{i<2}$ such that $\left(\pi_{c, n}\left(n_{i}, k_{i}, r_{i}\right)\right)_{i<2} \in L_{b, n}$ holds $\forall n \geq \max \left(n_{0}, n_{1}\right)$.

${ }^{1}$ Throughout the paper, the term acyclic will mean that there are no undirected cycles: that is, the graph $G \cup G^{-1}$ contains no cycles. 
Basic observations. Note that for any $c \in \mathbb{N}^{\mathbb{N}}$ and any odd $\aleph_{0}$-pair $b$, the definitions of $L_{c, n}, X_{c, n}$ and $L_{b, n}$ depend only on $(c(i))_{i \leq n}$ and $(b(i))_{i \leq n}$, respectively. For natural numbers $n^{\prime}>n$, define $\pi_{c, n, n^{\prime}}: X_{c, n^{\prime}} \cap\left\{(k, t) \mid t \in 2^{n^{\prime}-m}\right.$ for some $\left.m \leq n\right\} \rightarrow X_{c, n}$ by $\pi_{c, n, n^{\prime}}(k, t)=(k)-t \uparrow(n-m)$, where $m$ is chosen such that $t \in 2^{n^{\prime}-m}$. Observe that

$$
\pi_{c, n, n^{\prime}} \circ \pi_{c, n^{\prime}} \uparrow \operatorname{dom}\left(\pi_{c, n}\right)=\pi_{c, n}
$$

holds.

Let us use the abbreviation $E_{c}$ for $E_{\mathbb{L}_{c}}$. We list a number of useful basic observations about the family of digraphs and graphs defined above.

Claim 2.2. Assume that $b=(c, d)$ is an odd $\boldsymbol{\aleph}_{0}$-pair. Then

(1) $\mathbb{X}_{c}$ is a closed subset of $\mathbb{N} \times \mathbb{N} \times 2^{\mathbb{N}}$; hence it is a Polish space with the subspace topology.

(2) $\mathbb{L}_{c}=\mathbb{L}_{b} \cup \mathbb{L}_{b}^{-1}, L_{c, n}=L_{b, n} \cup L_{b, n}^{-1}$.

(3) If for some $n, k \in \mathbb{N}, \varepsilon<2, t \in 2^{<\mathbb{N}}$, we have $(k)^{\frown} t^{\frown}(\varepsilon) \in L_{c, n}$, then $(k)^{\frown} t^{\frown}(1-\varepsilon) \in L_{c, n}$ and $\operatorname{dist}^{L_{c, n}}\left((k)^{\frown} t^{\frown}(\varepsilon),(k)^{\frown} t^{\frown}(1-\varepsilon)\right)$ is odd.

(4) Let $(n, k, r),\left(n^{\prime}, k^{\prime}, r^{\prime}\right) \in \mathbb{X}_{c}$ with $n \leq n^{\prime}$. Then $(n, k, r) E_{c}\left(n^{\prime}, k^{\prime}, r^{\prime}\right)$ if and only if $r=t^{-} r^{*}$, $r^{\prime}=t^{\prime}-r^{*}$ with $|t|-\left|t^{\prime}\right|=n^{\prime}-n$ for some $r^{*} \in 2^{\mathbb{N}}, t, t^{\prime} \in 2^{<\mathbb{N}}$.

(5) $\mathbb{L}_{c}$ is acyclic and is 2-regular, except for a single vertex of degree 1.

(6) If $B \subseteq \mathbb{X}_{c}$ is Borel and meager, then so is $[B]_{E_{c}}$.

(7) If $B \subseteq \mathbb{X}_{c}$ is Borel and non-meager, then $\chi_{B}\left(\mathbb{L}_{c} \uparrow[B]_{E_{c}}\right)=3$.

(8) If $\lim \sup _{n} c(n)=\infty$, then $\mathbb{L}_{c}$ has the large gap property.

Proof. It is immediate from the definition of the graphs $\mathbb{L}_{b}$ that (1) and (2) hold, while (3) follows from the fact that $c \in(2 \mathbb{N}+1)^{\mathbb{N}}$.

To see (4), note that if $p=\left(x_{0}, \ldots, x_{l}\right)$ is an injective $\mathbb{L}_{c}$-walk, then, for large enough $m$, the sequence $\left(\pi_{c, m}\left(x_{0}\right), \ldots, \pi_{c, m}\left(x_{l}\right)\right)$ is an injective $L_{c, m}$-walk. It follows from the fact that the graphs $L_{c, m}$ are acyclic that $\operatorname{dist}^{\mathbb{L}_{c}}\left((n, k, r),\left(n^{\prime}, k^{\prime}, r^{\prime}\right)\right) \geq \operatorname{dist}^{L_{c, m}}\left(\pi_{c, m}(n, k, r), \pi_{c, m}\left(n^{\prime}, k^{\prime}, r^{\prime}\right)\right)$ for every large enough $m$. In particular, as the distance of the vertices in different copies of $L_{c, m-1}$ in $L_{c, m}$ is at least $c(m)+2$, we have that $(n, k, r) E_{c}\left(n^{\prime}, k^{\prime}, r^{\prime}\right)$ if and only if $\pi_{c, m}(n, k, r)$ and $\pi_{c, m}\left(n^{\prime}, k^{\prime}, r^{\prime}\right)$ are in the same copy of $L_{c, m-1}$ in $L_{c, m}$ for every large enough $m$, which is equivalent to the right-side condition in (4).

For (5), observe that for every $n$, every degree in $L_{c, n}$ is at most 2, and hence the same must be true for $\mathbb{L}_{c}$. Also, it is easy to see that if the degree of a vertex $(n, k, r) \in \mathbb{X}_{c}$ is $<2$, then for every large enough $n^{\prime}$, the degree of $\pi_{c, n^{\prime}}(n, k, r)$ in $L_{c, n^{\prime}}$ must be $<2$. It follows that this is possible only if $(n, k, r)=\left(0,0,(0)^{\mathbb{N}}\right)$. Finally, acyclicity follows from the acyclicity of $L_{c, n}$.

From (4), we get that $E_{c}$ is the union of the graphs of the partial maps and their inverses of the form

$$
f_{n, n^{\prime}, k, k^{\prime}, t, t^{\prime}}\left(n^{\prime}, k^{\prime}, t^{\prime} r\right)=\left(n, k, t^{\frown} r\right),
$$

where $n^{\prime} \geq n,|t|-\left|t^{\prime}\right|=n^{\prime}-n$. It is clear that the above partial maps are injective and preserve category. Thus,

$$
[B]_{E_{c}}=\bigcup_{n, n^{\prime}, k, k^{\prime}, t, t^{\prime}} f_{n, n^{\prime}, k, k^{\prime}, t, t^{\prime}}^{ \pm 1}(B)
$$

is also meager and Borel.

To see (7), first note that (5) implies $\chi_{B}\left(\mathbb{L}_{c} \uparrow[B]_{E_{c}}\right) \leq 3$ using the standard fact that the maximal vertex degree +1 is an upper bound [14, Proposition 4.6].

Assume that $B$ is a non-meager Borel set and $c:[B]_{E_{c}} \rightarrow 2$ is a Borel 2-coloring of $\mathbb{L}_{c} \uparrow[B]_{E_{c}}$. Then there exist an $i<2$ and a basic open set of the form $[(n, k, t)]\left(=\left\{(n, k, r) \in \mathbb{X}_{c} \mid r \sqsupset t\right\}\right)$, with $n, k \in \mathbb{N}$ and $t \in 2^{<\mathbb{N}}$, such that $[(n, k, t)] \backslash\left(B \cap c^{-1}(i)\right)$ is meager. Using (6), we have that $\left[[(n, k, t)] \backslash\left(B \cap c^{-1}(i)\right)\right]_{E_{c}}$ is also meager, so we can pick a point $(n, k, r) \in[(n, k, t)] \cap B \cap c^{-1}(i)$ 
that does not belong to this meager set. Assume that $r=t^{\frown}(\varepsilon)^{-} r^{\prime}$. Then, by (4), we have that $\left(n, k, t^{\frown}(1-\varepsilon)^{\frown} r^{\prime}\right) \in[(n, k, t)] \cap B \cap c^{-1}(i)$, so $c\left(n, k, t^{\frown}(\varepsilon)^{\frown} r^{\prime}\right)=c\left(n, k, t^{\frown}(1-\varepsilon)^{\frown} r^{\prime}\right)=i$. As in the proof of (4), it follows that

$$
\begin{gathered}
\operatorname{dist}^{\mathbb{L}_{c}}\left(\left(n, k, t \frown(\varepsilon) \frown r^{\prime}\right),\left(n, k, t^{\frown}(1-\varepsilon) \frown r^{\prime}\right)\right)= \\
\operatorname{dist}^{L_{c, n+|t|+1}}\left(\pi_{c, n+|t|+1}\left(n, k, \tau^{\frown}(\varepsilon)^{\frown} r^{\prime}\right), \pi_{c, n+|t|+1}\left(n, k, t \frown(1-\varepsilon)^{\frown} r^{\prime}\right)\right)= \\
\left.\operatorname{dist}^{L_{c, n+|t|+1}}\left((k) \frown t^{\frown}(\varepsilon)\right),(k)^{\frown} t^{\frown}(1-\varepsilon)\right),
\end{gathered}
$$

which is an odd number by (3). This contradicts the assumption that $c$ is a Borel 2-coloring of $\mathbb{L}_{B} \uparrow$ $[(n, k, r)]_{E_{c}} \subseteq \mathbb{L}_{B} \uparrow[B]_{E_{c}}$.

Finally, for (8), it is easy to verify that $B=\left\{(0,0, r) \in \mathbb{X}_{c} \mid r \in 2^{\mathbb{N}}\right\}$ witnesses the large gap property for $\mathbb{L}_{c}$ whenever $\lim \sup _{n} c(n)=\infty$.

Claim 2.3. Assume that $L, L^{\prime}$ are $\leq 2$-regular acyclic Borel graphs on standard Borel spaces $X, X^{\prime}$.

(1) Let $B$ be an L-invariant Borel set so that $E_{L} \uparrow B$ is smooth. Then $\chi_{B}(L \uparrow B) \leq 2$.

(2) Assume that $\phi$ is a Borel homomorphism from $L$ to $L^{\prime}$. Define $M=\left\{x \in X \mid[\phi(x)]_{E_{L^{\prime}}} \backslash \phi\left([x]_{E_{L}}\right) \neq\right.$ $\emptyset\}$. Then $M$ is Borel and $\chi_{B}(L \uparrow M) \leq 2$.

Proof. To see (1), note that $E_{L}$ is countable, so smoothness is equivalent to the existence of an $L$ invariant Borel partial mapping $x \rightarrow y_{x}$ so that $y_{x} E_{L} x$ holds for every $x \in B$. Clearly the map $c: B \rightarrow 2$, defined by $c(x)=0$ iff $\operatorname{dist}^{L}\left(x, y_{x}\right)$ is even, is a Borel 2-coloring of the graph $L \uparrow B$.

For (2), first note that the Luzin-Novikov theorem yields that $M$ is Borel. Fix a Borel linear ordering < of $X$. Since $L^{\prime} \uparrow \phi\left([x]_{E_{L}}\right)$ and $L^{\prime} \uparrow[\phi(x)]_{E_{L^{\prime}}}$ are $\leq 2$-regular acyclic connected graphs, there are one or two vertices in $[\phi(x)]_{E_{L^{\prime}}} \backslash \phi\left([x]_{E_{L}}\right)$ that have an $L^{\prime}$-neighbor in $\phi\left([x]_{E_{L}}\right)$. Let $y_{x}$ be the <-minimal such vertex. Now, as in (1), letting $c(x)=0$ iff $\operatorname{dist}^{L^{\prime}}\left(\phi(x), y_{x}\right)$ is even, it follows that $\chi_{B}(L \uparrow M) \leq 2$.

The following claim will be used to establish Theorem 1.1 for Borel graphs.

Claim 2.4. Assume that $G$ is a Borel graph on a standard Borel space $X, c \in(2 \mathbb{N}+1)^{\mathbb{N}}$ and $\left(\phi_{n}\right)_{n \in \mathbb{N}}$ is a sequence of Borel partial maps from $X$ to $X_{c, n}$ with the following properties for every $n \in \mathbb{N}$ :

(1) $\operatorname{dom}\left(\phi_{n}\right) \subseteq \operatorname{dom}\left(\phi_{n+1}\right)$ and $\bigcup_{n \in \mathbb{N}} \operatorname{dom}\left(\phi_{n}\right)=X$.

(2) The map $\phi_{n}$ is a partial homomorphism from $G$ to $L_{c, n}$.

(3) $\phi_{n}=\pi_{c, n, n+1} \circ \phi_{n+1} \uparrow \operatorname{dom}\left(\phi_{n}\right)$.

Then there exists a Borel homomorphism $\phi$ from $G$ to $\mathbb{L}_{c}$.

Moreover,

(4) If $X$ is Polish, for every $n \in \mathbb{N}$, the set $\operatorname{dom}\left(\phi_{n}\right)$ is open in $X$, and the maps $\phi_{n}$ are continuous,

then $\phi$ can be chosen to be continuous.

Proof. Let $x \in X$ be arbitrary, and take $n_{0}^{x}$ to be minimal such that $x \in \operatorname{dom}\left(\phi_{n_{0}}\right)$. For $n \geq n_{0}$, we have that $\phi_{n}(x)=\left(k_{n}\right)^{-} t_{n}$ for some $t_{n} \in 2^{n-m_{n}}$ and $k_{n}, m_{n} \in \mathbb{N}$. By (3), for every $n \geq n_{0}$, the relations $k_{n}=k_{n+1}, m_{n}=m_{n+1}$ and $t_{n} \sqsubset t_{n+1}$ hold. Let $\phi(x)=\left(m_{n_{0}}, k_{n_{0}}, \bigcup_{n \geq n_{0}} t_{n}\right)$. Clearly, $\phi$ is a Borel map; we will check that it is a homomorphism. Indeed, if $\left(x_{i}\right)_{i<2} \in G$, then, by (2), letting $n \geq \max \left\{n_{0}^{x_{i}} \mid i<2\right\}$, we have that $\left(\phi_{n}\left(x_{i}\right)\right)_{i<2} \in L_{c, n}$. Notice that $\pi_{c, n}(\phi(x))=\phi_{n}(x)$ whenever $x \in \operatorname{dom}\left(\phi_{n}\right)$, so we obtain $\left(\pi_{c, n}\left(\phi\left(x_{i}\right)\right)\right)_{i<2}=\left(\phi_{n}\left(x_{i}\right)\right)_{i<2} \in L_{c, n}$, which verifies our claim by the definition of $\mathbb{L}_{c}$.

Finally, one can easily check that the assumptions in (4) of the claim yield the continuity of $\phi$. 


\section{A basis under continuous reducibility}

In this section, we construct a basis for Borel digraphs with Borel chromatic number $>2$. We will show these results in somewhat greater generality than stated in the introduction, namely for analytic graphs defined on Hausdorff spaces. The proof relies on a slight modification of the idea behind the $\mathbb{G}_{0}$ dichotomy, together with an observation about the Borel 2-colorability of Borel digraphs, which is essentially summarized in Claims 3.3, 3.4 and 3.5 below.

Theorem 3.1. Let $G$ be an analytic digraph on a Hausdorff space X. Then exactly one of the following holds:

(1) $\chi_{B}(G) \leq 2$.

(2) There exists an odd $\boldsymbol{\aleph}_{0}$-pair $b$ so that $\mathbb{L}_{b}$ admits a continuous homomorphism to G. Moreover, for any $f \in \mathbb{N}^{\mathbb{N}}$, the pair $b=(c, d)$ can be chosen in such a way that, for every $i \in \mathbb{N}$, we have $\Sigma(d(i))>f(i) \cdot \sum_{j<i}|\Sigma(d(j))|$.

Proof. The proof will follow the proof of the $\mathbb{G}_{0}$ dichotomy presented in [20].

Fix a function $f \in \mathbb{N}^{\mathbb{N}}$. As $G$ is analytic, there exist a continuous surjection $\phi_{G}: \mathbb{N}^{\mathbb{N}} \rightarrow G$ and a continuous map $\phi_{X}: \mathbb{N}^{\mathbb{N}} \rightarrow X$ such that $\phi_{X}\left(\mathbb{N}^{\mathbb{N}}\right)$ is the union of the two projections of $G$ to $X$. By iteratively throwing away $G$-invariant sets restricted to which $G$ has a Borel 2-coloring, we will define a decreasing sequence $\left(X^{\alpha}\right)_{\alpha<\omega_{1}}$ of analytic subsets of $X$. Let $X^{0}=\phi_{X}\left(\mathbb{N}^{\mathbb{N}}\right)$ and $X^{\lambda}=\bigcap_{\alpha<\lambda} X^{\alpha}$ if $\lambda$ is a limit ordinal.

Let us now describe the successor stage.

An approximation is a quadruple $a=\left(n^{a}, b^{a}, \phi^{a}, \psi^{a}\right)$, where $n^{a} \in \mathbb{N}, b^{a}=\left(c^{a}, d^{a}\right)$ is an odd $n^{a}$ -

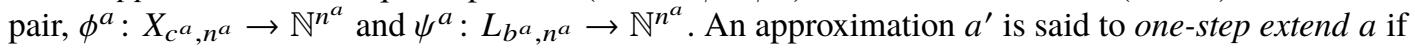

(a) $n^{a^{\prime}}=n^{a}+1$.

(b) $c^{a^{\prime}} \sqsupset c^{a}, d^{a^{\prime}} \sqsupset d^{a}$.

(c) $\Sigma\left(d^{a^{\prime}}\left(n^{a^{\prime}}\right)\right)>f\left(n^{a}\right) \cdot \sum_{j \leq n^{a}}\left|\Sigma\left(d^{a}(j)\right)\right|$.

(d) $\forall x \in \operatorname{dom}\left(\pi_{c^{a^{\prime}}, n^{a}, n^{a^{\prime}}}\right) \phi^{a^{\prime}}(x) \sqsupset \phi^{a} \circ \pi_{c^{a^{\prime}}, n^{a}, n^{a^{\prime}}}(x)$.

(e) $\forall x, y \in \operatorname{dom}\left(\pi_{c^{a^{\prime}}, n^{a}, n^{a^{\prime}}}\right)$

$$
(x, y) \in L_{b^{a^{\prime}}, n^{a^{\prime}}} \Longrightarrow \psi^{a^{\prime}}(x, y) \sqsupset \psi^{a}\left(\pi_{c^{a^{\prime}}, n^{a}, n^{a^{\prime}}}(x), \pi_{c^{a^{\prime}}, n^{a}, n^{a^{\prime}}}(y)\right) .
$$

A configuration is a quadruple of the form $\gamma=\left(n^{\gamma}, b^{\gamma}, \phi^{\gamma}, \psi^{\gamma}\right)$, where $n^{\gamma} \in \mathbb{N}, b^{\gamma}$ is an odd $n^{\gamma}$-pair, $\phi^{\gamma}: X_{c^{\gamma}, n^{\gamma}} \rightarrow \mathbb{N}^{\mathbb{N}}$ and $\psi^{\gamma}: L_{b^{\gamma}, n^{\gamma}} \rightarrow \mathbb{N}^{\mathbb{N}}$ has the following property: for every $(x, y) \in L_{b^{\gamma}, n^{\gamma}}$,

$$
\left(\phi_{G} \circ \psi^{\gamma}\right)(x, y)=\left(\phi_{X} \circ \phi^{\gamma}(x), \phi_{X} \circ \phi^{\gamma}(y)\right) .
$$

A configuration $\gamma$ is said to be compatible with an approximation $a$ if

(1) $n^{a}=n^{\gamma}$.

(2) $b^{a}=b^{\gamma}$.

(3) $\forall x \in X_{c^{\gamma}, n^{\gamma}} \phi^{a}(x) \sqsubset \phi^{\gamma}(x)$.

(4) $\forall(x, y) \in L_{b^{\gamma}, n^{\gamma}} \psi^{a}(x, y) \sqsubset \psi^{\gamma}(x, y)$.

We say that a configuration $\gamma$ is compatible with a set $Y \subseteq X$ if $\phi_{X} \circ \phi^{\gamma}\left(X_{c^{\gamma}, n^{\gamma}}\right) \subseteq[Y]_{E_{G}}$. An approximation $a$ is $Y$-terminal if no configuration is compatible with both $Y$ and a one-step extension of $a$. Let

$$
A(a, Y)=\left\{\phi_{X} \circ \phi^{\gamma}\left(s_{n^{\gamma}}\right) \mid \gamma \text { is compatible with } a \text { and } Y\right\}
$$

Lemma 3.2. Suppose that $Y \subseteq X$ is an analytic set and $a$ is a $Y$-terminal approximation. Then there exists a $G$-invariant Borel set $B(a, Y) \supseteq[A(a, Y)]_{E_{G}}$ so that $G \uparrow B(a, Y)$ has a Borel 2-coloring, $c_{a, Y}$.

We start with a series of claims. 
Claim 3.3. Suppose that $A \subseteq X$ is an analytic set such that for every $x, y \in A$, every $G$-walk from $x$ to $y$ has even (undirected or, equivalently, directed) length. Then there exists a $G$-invariant Borel set $B \supseteq[A]_{E_{G}}$ on which $G \uparrow B$ admits a Borel 2-coloring.

Proof. For $i<2$, define $A_{i} \subseteq[A]_{E_{G}}$ as follows: let $x \in A_{i}$ if there exists a walk of length $n$ from $x$ to some $y \in A$, where $n \equiv i \bmod 2$. It is clear that the sets $A_{i}$ are analytic, their union covers $[A]_{E_{G}}$ and they are $G$-independent. Note that their $G$-independence implies that $A_{0} \cap A_{1}=\emptyset$. By the separation theorem for analytic sets, there exist Borel sets $B_{i} \supseteq A_{i}$ with $B_{0} \cap B_{1}=\emptyset$. Define $c(x)=i \Longleftrightarrow x \in B_{i}$, and let $C=\left\{x \in X \mid c\right.$ is a 2-coloring of $\left.G \uparrow[x]_{E_{G}}\right\}$. Clearly, the sets $X \backslash C$ and $A_{0} \cup A_{1}$ are disjoint, analytic and $G$-invariant. Hence, by [11, Lemma 5.1], there exists a $G$-invariant Borel set $B \supseteq A_{0} \cup A_{1}$ with $B \cap(X \backslash C)=\emptyset$. Then $c \uparrow B$ is a Borel 2-coloring of $G \uparrow B$.

Claim 3.4. Let $A^{\prime} \subseteq A \subseteq X$ be analytic sets and $d \in \mathbb{Z}$ be an odd number. Assume that, for every $x^{\prime} \in A^{\prime}$, there exists an $x \in A$ such that there exists a $G$-walk from $x^{\prime}$ to $x$ of directed length $d$. Moreover, assume that every odd length $G$-walk between elements of $A$ has directed length $\leq|d|$. Then there exists a $G$-invariant Borel set $B \supseteq\left[A^{\prime}\right]_{E_{G}}$ on which $G \uparrow B$ admits a Borel 2-coloring.

Proof. Suppose that $d>0$; the other case is analogous. Let $A_{0}^{\prime}=\left\{x^{\prime} \in A^{\prime} \mid\right.$ there exists a $G$-walk from $x^{\prime}$ to some element of $A$ with odd negative directed length\}. We claim that $A_{0}^{\prime}$ satisfies the assumptions of Claim 3.3. Assume that this is not the case: that is, there exists $x^{\prime}, y^{\prime} \in A_{0}^{\prime}$ so that there exists a $G$ walk of odd length between $x^{\prime}$ and $y^{\prime}$. As the directed length of an odd length walk is non-zero, we can assume (switching the roles of $x^{\prime}$ and $y^{\prime}$ if necessary) that there exists a walk $p$ from $x^{\prime}$ to $y^{\prime}$ of positive odd directed length. Then, using our assumptions on $A_{0}^{\prime}$ and $A^{\prime}$, there exist $w, z \in A$ and $G$-walks $q, r$ such that $q$ is a walk from $w$ to $x^{\prime}, r$ is a walk from $y^{\prime}$ to $z$ and $\operatorname{dilength}(q)>0$, dilength $(r)=d$ and both

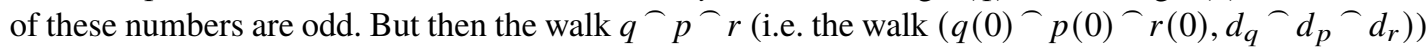
connects $w$ with $z$ and dilength $\left(q^{\frown} p^{\frown} r\right)>d+\operatorname{dilength}(p)$, and the former is an odd number $>d$, contradicting our assumption on $A$.

Now let $B_{0}$ be the invariant Borel superset of $\left[A_{0}^{\prime}\right]_{E_{G}}$ provided by Claim 3.3, and define $A_{1}^{\prime}=A^{\prime} \backslash B_{0}$. Clearly, by the definition of $A_{0}^{\prime}$ and as $A_{1}^{\prime} \subseteq A$, the set $A_{1}^{\prime}$ also satisfies the requirements of Claim 3.3, so let $B_{1} \supseteq\left[A_{1}^{\prime}\right]_{E_{G}}$ be the Borel set it yields. Then it is easy to see, from the invariance of $B_{0}$ and $B_{1}$, that $B=B_{0} \cup B_{1}$ satisfies the requirements of the claim.

Claim 3.5. Let $A \subseteq X$ be an analytic set, and assume that there exists an $n \in \mathbb{N}$ such that if $x, y \in A$ and $p$ is a $G$-walk of odd length from $x$ to $y$, then dilength $(p) \leq n$. Then there exists a $G$-invariant Borel set $B \supseteq[A]_{E_{G}}$ such that $G \uparrow B$ admits a Borel 2-coloring.

Proof. We prove this statement by induction on the minimal $n$ with this property. If $n=0$, then Claim 3.3 yields the required conclusion.

Now assume that we have shown the statement for every natural number $\leq n-1$. If $n>0$ is even, then it cannot be minimal, and hence there is nothing to show. So we can assume that $n$ is odd. For $\varepsilon \in\{-1,1\}$, let $A_{n, \varepsilon}=\{x \in A$ : there exists a $G$-walk from $x$ to some $y \in A$ of directed length $\varepsilon \cdot n\}$. Now we can apply Claim 3.4 to the sets $A_{n, \varepsilon}, A$ and $\varepsilon \cdot n$. This yields $G$-invariant Borel sets $B_{\varepsilon} \supseteq A_{n, \varepsilon}$ on which $G$ admits a Borel 2-coloring. Note that if $x \in A \backslash\left(B_{-1} \cup B_{1}\right)$, then every odd length walk between $x$ and an element of $A$ must have directed length $<n$. So, by the inductive hypothesis, we can find an invariant Borel set $B^{n-1} \supseteq\left[A \backslash\left(B_{-1} \cup B_{1}\right)\right]_{E_{G}}$ such that $G \uparrow B$ admits a Borel 2-coloring. Using the invariance of $B^{n-1}, B_{-1}$, and $B_{1}$ again, we obtain that $G \uparrow B^{n-1} \cup B_{-1} \cup B_{1}$ also admits a Borel 2-coloring, which finishes the proof.

Proof of Lemma 3.2. By definition, the set $A(a, Y)$ is analytic. If there exists an $n \in \mathbb{N}$ such that every walk $p$ of odd length between vertices from $A(a, Y)$ has directed length $\leq n$, then Claim 3.5 yields the $G$-invariant Borel set $B(a, Y) \supseteq[A(a, Y)]_{E_{G}}$ and a Borel 2-coloring $c_{a, Y}$ of $G \uparrow B(a, Y)$.

So assume that such an $n$ does not exist; we will show that $a$ is not $Y$-terminal. Using this assumption for $n=f\left(n^{a}\right) \cdot \sum_{j \leq n^{a}}\left|\Sigma\left(d^{a}(j)\right)\right|$, we obtain two configurations $\left(\gamma_{j}\right)_{j<2}$ compatible with $a$ and $Y$, a 
$G$-walk of odd length $p=\left(\left(x_{0}, \ldots, x_{m+2}\right), d_{p}\right)$ with dilength $(p)>f\left(n^{a}\right) \cdot \sum_{j \leq n^{a}}\left|\Sigma\left(d^{a}(j)\right)\right|$ such that $x_{0}=\left(\phi_{X} \circ \phi^{\gamma_{0}}\right)\left(s_{n^{a}}\right)$ and $x_{l}=\left(\phi_{X} \circ \phi^{\gamma_{1}}\right)\left(s_{n^{a}}\right)$. Pick $r_{0}, \ldots, r_{m+2} \in \mathbb{N}^{\mathbb{N}}$ and $e_{0}, \ldots, e_{m+1} \in \mathbb{N}^{\mathbb{N}}$ so that

$\circ r_{0}=\phi^{\gamma_{0}}\left(s_{n^{a}}\right), r_{m+2}=\phi^{\gamma_{1}}\left(s_{n^{a}}\right)$,

$\circ \forall j \leq m+2 \phi_{X}\left(r_{j}\right)=x_{j}$, and

○ $\forall j<m+2 \phi_{G}\left(e_{j}\right)=\left(x_{j}, x_{j+1}\right)^{d_{p}(j)}$.

We define a configuration $\delta$ as follows: let $n^{\delta}=n^{a}+1$ and $b^{\delta}=\left(c^{\delta}, d^{\delta}\right)=\left(c^{a} \frown m, d^{a} \frown d_{p}\right)$, and define $\phi^{\delta}: X_{c^{\delta}, n^{\delta}} \rightarrow \mathbb{N}^{\mathbb{N}}$ by

$$
\begin{cases}\phi^{\delta}(x-(j))=\phi^{\gamma_{j}}(x) & \text { for } x \in X_{c^{a}, n^{a}} \text { and } j<2, \text { and } \\ \phi^{\delta}((j))=r_{j+1} & \text { for } j \leq m .\end{cases}
$$

Finally, define $\psi^{\delta}: L_{b^{\delta}, n^{\delta}} \rightarrow \mathbb{N}^{\mathbb{N}}$ by

$$
\begin{cases}\psi^{\delta}\left(x-(j), y^{-}(j)\right)=\psi^{\gamma^{j}}(x, y) & \text { for }(x, y) \in L_{b^{a}, n^{a}} \text { and } j<2 . \\ \psi^{\delta}\left(\left(s_{n^{a}}-(0),(0)\right)^{d(0)}\right)=e_{0} . & \\ \psi^{\delta}\left(\left((m), s_{n^{a}}-(1)\right)^{d(m+1)}\right)=e_{m+1} . & \\ \psi^{\delta}\left((j, j+1)^{d(j+1)}\right)=e_{j+1} & \text { for } j \leq m-1 .\end{cases}
$$

It is not hard to check that $\delta$ is a configuration. Moreover, as $\gamma_{0}$ and $\gamma_{1}$ are compatible with $Y$, so is $\delta$. Finally, using the fact that $\Sigma(d)=\operatorname{dilength}(p)>f\left(n^{a}\right) \cdot \sum_{j \leq n^{a}}\left|\Sigma\left(d^{a}(j)\right)\right|$, one can deduce that there exists a unique one-step extension $a^{\prime}$ of $a$ that is compatible with $\delta$. This contradicts the assumption that $a$ was $Y$-terminal.

Define

$$
X^{\alpha+1}=X^{\alpha} \backslash \underset{a \text { is } X^{\alpha} \text {-terminal, }}{\bigcup} B\left(a, X^{\alpha}\right) .
$$

Since there are only countably many possible approximations, and $X^{0}$ is an analytic set, the set $X^{\alpha}$ is analytic for every $\alpha<\omega_{1}$. Note also that each $X^{\alpha}$ is $G$-invariant.

Lemma 3.6. Assume that $\alpha<\omega_{1}$ and $a$ is an approximation that is not $X^{\alpha+1}$-terminal. Then a has a one-step extension that is not $X^{\alpha}$-terminal.

Proof. Let $a^{\prime}$ be a one-step extension of $a$ for which there exists a configuration $\gamma$ compatible with $X^{\alpha+1}$ and $a^{\prime}$. Then $\emptyset \neq\left(\phi_{X} \circ \phi^{\gamma}\right)\left(X_{c^{\gamma}, n^{\gamma}}\right) \subseteq\left[X^{\alpha+1}\right]_{E_{G}}=X^{\alpha+1}$; but if $a^{\prime}$ was $X^{\alpha}$-terminal, then $\left[\left(\phi_{X} \circ \phi^{\gamma}\right)\left(X_{c^{\gamma}, n^{\gamma}}\right)\right]_{E_{G}} \subseteq\left[A\left(a^{\prime}, X^{\alpha}\right)\right]_{E_{G}}$ would be covered by $B\left(a^{\prime}, X^{\alpha}\right)$, contradicting the definition and the $G$-invariance of $X^{\alpha+1}$.

Note that the set of $X^{\alpha}$-terminal approximations increases as $\alpha$ increases, and there are only countably many approximations. Thus we can fix an $\alpha<\omega_{1}$ so that the $X^{\alpha}$-terminal and $X^{\alpha+1}$-terminal approximations are the same.

Lemma 3.7. If every approximation is $X^{\alpha+1}$-terminal, then $G$ has a Borel 2-coloring.

Proof. Observe first that $X^{\alpha+1}$ is $G$-independent: otherwise, if $(x, y) \in G \cap\left(X^{\alpha+1}\right)^{2}$, then there exists a configuration $\gamma$ with $c^{\gamma}=(1)$ compatible with $\{x, y\}$. Consequently, there exists an approximation $a$ that is compatible with $\gamma$ and $X^{\alpha+1}$. Then $a$ is $X^{\alpha+1}$-terminal, so $x, y \in\left[A\left(a, X^{\alpha+1}\right)\right]_{E_{G}}$, but then $a$ is an $X^{\alpha}$-terminal approximation as well, so $x, y \in\left[A\left(a, X^{\alpha}\right)\right]_{E_{G}} \subseteq B\left(a, X^{\alpha}\right)$, contradicting the definition of $X^{\alpha+1}$

Moreover, $X^{\alpha+1} \subseteq X^{0}$ is $G$-independent and $G$-invariant, so, by the definition of $X^{0}$, it must be empty. 
Let $e:\left\{(a, \beta): a\right.$ is $X^{\beta}$ terminal, $\left.\beta \leq \alpha\right\} \rightarrow \mathbb{N}$ be an injection and let $c_{a, X^{\beta}}$ be the Borel 2-coloring of $G \uparrow B\left(a, X^{\beta}\right)$ given by Lemma 3.2, for $(a, \beta) \in \operatorname{dom}(e)$. If $x \in X$, define

$$
c(x)= \begin{cases}c_{a, X^{\beta}}(x) & \text { if } e(a, \beta) \text { is minimal such that } x \in B\left(a, X^{\beta}\right), \text { and } \\ 0 & \text { if } x \notin \bigcup_{(a, \beta) \in \operatorname{dom}(e)} B\left(a, X^{\beta}\right) .\end{cases}
$$

It is easy to check that $c$ is a Borel map defined on $X$, while the $G$-invariance of the sets $B\left(a, X^{\beta}\right)$ implies that $c$ is a 2-coloring.

Now we are ready to finish the proof of Theorem 3.1. Assume $\chi_{B}(G)>2$. Then, by Lemma 3.7 , there exists an approximation that is not $X^{\alpha+1}$-terminal. Clearly we can find such an $a_{0}$ with $n^{a_{0}}=0$. By applying Lemma 3.6 recursively, we obtain one-step extensions $a_{n+1}$ of $a_{n}$ that are not $X^{\alpha}$-terminal, with $n^{a_{n}}=n$. Define $b=(c, d)=\bigcup_{n} b^{a_{n}}, \phi: \mathbb{X}_{c} \rightarrow \mathbb{N}^{\mathbb{N}}$, and $\psi: \mathbb{L}_{b} \rightarrow \mathbb{N}^{\mathbb{N}}$ by letting $\phi(m, k, r)=\bigcup_{n \geq m} \phi^{a_{n}}\left(\pi_{c, n}(m, k, r)\right)$ and, for $\left(m_{i}, k_{i}, r_{i}\right)_{i<2} \in \mathbb{L}_{b}, \psi\left(\left(m_{i}, k_{i}, r_{i}\right)_{i<2}\right)=$ $\bigcup_{n \geq m_{0}, m_{1}} \psi^{a_{n}}\left(\left(\pi_{c, n}\left(m_{i}, k_{i}, r_{i}\right)\right)_{i<2}\right)$. It follows from the fact that $a_{n+1}$ one-step extends $a_{n}$ (using conditions (d) (e), and the fact that $\left.\pi_{c, n, n^{\prime}} \circ \pi_{c, n^{\prime}} \uparrow \operatorname{dom}\left(\pi_{c, n}\right)=\pi_{c, n}\right)$ that $\phi$ and $\psi$ are well-defined.

Now we check that $\phi_{X} \circ \phi$ is a continuous homomorphism from $\mathbb{L}_{b}$ to $G$. The continuity of this mapping is clear from its definition; we check that it is a homomorphism. To this end, let $\left(x_{0}, x_{1}\right) \in \mathbb{L}_{b}$ with $x_{i}=\left(n_{i}, k_{i}, r_{i}\right)$, for $i<2$. We claim that

$$
\left(\phi_{G} \circ \psi\right)\left(x_{0}, x_{1}\right)=\left(\left(\phi_{X} \circ \phi\right)\left(x_{0}\right),\left(\phi_{X} \circ \phi\right)\left(x_{1}\right)\right),
$$

which is clearly sufficient, as the left side is an element of $G$. We show that if $U$ and $V$ are open neighborhoods of $\left(\phi_{G} \circ \psi\right)\left(x_{0}, x_{1}\right)$ and $\left(\left(\phi_{X} \circ \phi\right)\left(x_{0}\right),\left(\phi_{X} \circ \phi\right)\left(x_{1}\right)\right)$, then $U \cap V \neq \emptyset$.

By the definition of $\mathbb{L}_{b}$, we have that $\left(\pi_{c, n}\left(x_{i}\right)\right)_{i<2} \in L_{b, n}$ for every $n \geq \max \left(n_{0}, n_{1}\right)$. Thus, using the continuity of $\phi, \psi, \phi_{G}$ and $\phi_{X}$, we can find an $n \geq \max \left(n_{0}, n_{1}\right)$ so large that $U \supseteq$ $\phi_{G}\left(\left[\psi^{a_{n}}\left(\left(\pi_{c, n}\left(x_{i}\right)\right)_{i<2}\right)\right]\right)$ and $V \supseteq \phi_{X}\left(\left[\psi^{a_{n}} \circ \pi_{c, n}\left(x_{0}\right)\right]\right) \times \phi_{X}\left(\left[\phi^{a_{n}} \circ \pi_{c, n}\left(x_{1}\right)\right]\right)$.

Let $\gamma$ be a configuration compatible with $a_{n}$. Then, by (3.1), we have that

$$
\left(\phi_{G} \circ \psi^{\gamma}\right)\left(\left(\pi_{c, n}\left(x_{i}\right)\right)_{i<2}\right)=\left(\left(\phi_{X} \circ \phi^{\gamma}\right)\left(\pi_{c, n}\left(x_{0}\right)\right),\left(\phi_{X} \circ \phi^{\gamma}\right)\left(\pi_{c, n}\left(x_{1}\right)\right) .\right.
$$

It then follows from the compatibility of $\gamma$ and $a_{n}$ that

$$
\left(\left(\phi_{X} \circ \phi^{\gamma}\right)\left(\pi_{c, n}\left(x_{0}\right)\right),\left(\phi_{X} \circ \phi^{\gamma}\right)\left(\pi_{c, n}\left(x_{1}\right)\right)\right) \in \phi_{X}\left(\left[\phi^{a_{n}} \circ \pi_{c, n}\left(x_{0}\right)\right]\right) \times \phi_{X}\left(\left[\phi^{a_{n}} \circ \pi_{c, n}\left(x_{1}\right)\right]\right) \subseteq V
$$

and

$$
\phi_{G} \circ \psi^{\gamma}\left(\left(\pi_{c, n}\left(x_{i}\right)\right)_{i<2}\right) \in \phi_{G}\left(\left[\psi^{a_{n}}\left(\left(\pi_{c, n}\left(x_{i}\right)\right)_{i<2}\right)\right]\right) \subseteq U,
$$

which together with (3.3) implies $U \cap V \neq \emptyset$, finishing the proof of Theorem 3.1.

\section{Large gaps}

In this section, we complete the proof of Theorem 1.1 and prove Theorem 1.2. Note that graphs (rather than digraphs) will be considered. Let $L$ be a graph on the space $X$, and assume that $B \subset X$. The minimal cardinality of an $L \uparrow X \backslash B$-component will be denoted by $\operatorname{mgs}(B)$.

We start with an easy observation.

Claim 4.1. Let $L$ be a $\leq$ 2-regular acyclic Borel graph on a standard Borel space $X$ that has the large gap property. Then there exists an increasing sequence $\left(B_{n}\right)_{n \in \mathbb{N}}$ of Borel subsets of $X$ such that $\bigcup_{n \in \mathbb{N}} B_{n}$ is L-invariant, $E_{L} \uparrow X \backslash \bigcup_{n \in \mathbb{N}} B_{n}$ is smooth, for every $n \in \mathbb{N}$ the $L \uparrow B_{n}$-components are finite, and $\operatorname{mgs}\left(B_{n}\right) \rightarrow \infty$ as $n \rightarrow \infty$. 
Proof. Let $B$ be a Borel set witnessing the large gap property for $L$. The graph $L$ restricted to an $L$-component is an infinite, connected $\leq 2$-regular graph that can be partitioned into disjoint $L \uparrow X \backslash B$ components. Let $S_{0}$ be the union of those $L$-components

○ That contain an infinite $L \uparrow X \backslash B$-component, or

- For which the lim sup of the cardinality of the $L \uparrow X \backslash B$-components is finite in one of the directions.

It follows from the choice of $B$ that $S_{0}$ is Borel and $E_{L} \uparrow S_{0}$ is smooth. Let

$$
B_{n}=B \cup\left\{x \in X \backslash S_{0}: \text { the } L \uparrow X \backslash B \text {-component of } x \text { has size }<n\right\} .
$$

Clearly the sets $B_{n}$ are increasing, $X \backslash S_{0}=\bigcup_{n \in \mathbb{N}} B_{n}$ and $\operatorname{mgs}\left(B_{n}\right) \geq n$. Finally, note that if the $L \uparrow B_{n^{-}}$ component of $x$ is infinite, then the cardinality of the $L \uparrow X \backslash B$-components would be bounded by $n$ in some of the directions in the $L$-connected component of $x$ : that is, $x \in S_{0}$.

The next proposition is the essence of the argument.

Proposition 4.2. Assume that $c \in(2 \mathbb{N}+1)^{\mathbb{N}}$.

(1) Let L be a $\leq$-regular acyclic Borel graph on the standard Borel space X. Assume that $\left(B_{n}\right)_{n \in \mathbb{N}}$ is an increasing sequence of Borel subsets of $X$ with $\bigcup_{n \in \mathbb{N}} B_{n}=X$ and $\operatorname{mgs}\left(B_{n}\right) \rightarrow \infty$, and for every $n$, the $L \uparrow B_{n}$-components are finite. Then $L \leq_{B} \mathbb{L}_{c}$.

(2) If $c^{0} \in(2 \mathbb{N}+1)^{\mathbb{N}}$ and $c^{0}(n) \rightarrow \infty$, then $\mathbb{L}_{c^{0}} \leq_{c} \mathbb{L}_{c}$.

Our strategy is to inductively define sequences $k_{n} \in \mathbb{N}$ and $\phi_{n}: B_{k_{n}} \rightarrow X_{c, n}$ and appeal to Claim 2.4. We start with the key lemma.

\section{Lemma 4.3.}

(1) Let $B \subseteq B^{\prime} \subseteq X$ be Borel sets for which every component of $L \uparrow B$ is finite, $n$ be a natural number for which $\operatorname{mgs}(B)>2 \cdot \operatorname{length}\left(L_{c, n+1}\right)$, and $\phi$ be a Borel homomorphism from $L \uparrow B$ to $L_{c, n}$. Then there exists a homomorphism $\phi^{\prime}$ from $L \uparrow B^{\prime}$ to $L_{c, n+1}$ so that $\pi_{c, n, n+1} \circ \phi^{\prime} \uparrow B=\phi$.

(2) Moreover, if $L=\mathbb{L}_{c^{0}}, B=\left\{(l, m, r) \in \mathbb{X}_{c^{0}}: l<k\right\}, B^{\prime}=\left\{(l, m, r) \in \mathbb{X}_{c^{0}}: m<k^{\prime}\right\}$ for $k<k^{\prime}$, and $\phi$ is continuous, then $\phi^{\prime}$ can be taken to be continuous.

Proof. First we show (1). Note that the graph $L \uparrow B^{\prime}$ is a disjoint union of finite walks. Fix a Borel linear ordering $<$ of $X$. We will define $\phi^{\prime}$ so that the value $\phi^{\prime}(x)$ will only depend on

(a) The values of $\phi$ on the $L \uparrow B^{\prime}$-component of $x$, and

(b) The index of $x$ in the unique enumeration $\left(v_{i}^{x}\right)_{i \leq m^{x}}$ of the $L \uparrow B^{\prime}$-component of $x$ with the property that $v_{0}^{x}<v_{m^{x}}^{x}$ and $\forall i<m^{x}$, we have $\left(v_{i}^{x}, v_{i+1}^{x}\right) \in L$.

Claim 4.4. For a connected component of $L \uparrow B^{\prime}$, let $\left(v_{i}\right)_{i<m}$ be the enumeration described in $(b)$. There exists a homomorphism $\psi$ of $L \uparrow\left\{v_{0}, \ldots, v_{m}\right\}$ to $L_{c, n+1}$ so that we have $\pi_{c, n, n+1} \circ \psi \uparrow\left\{v_{0}, \ldots, v_{m}\right\} \cap B=$ $\phi \uparrow\left\{v_{0}, \ldots, v_{m}\right\} \cap B$.

Proof of Claim 4.4. To see that such a homomorphism $\psi$ exists, note that the set $\left\{v_{0}, \ldots, v_{m}\right\}$ decomposes into connected components of $L \uparrow B$ and walks connecting them: more precisely, there are an odd number $l$ and a sequence $0 \leq i_{0}<i_{1}<\cdots<i_{l} \leq m$ with the property that if $i \in\left[0, i_{0}\right) \cup\left(i_{1}, i_{2}\right) \cup\left(i_{3}, i_{4}\right) \cup \cdots \cup\left(i_{l}, m\right]$ (where the first and last intervals could be empty), then $v_{i} \in B^{\prime} \backslash B$, while for every even $j<l,\left\{v_{i_{j}}, \ldots, v_{i_{j+1}}\right\}$ is an $L \uparrow B$-component.

Define $\psi\left(v_{i}\right)$ for $i \in\left[i_{0}, \ldots, i_{1}\right]$ to be $\phi\left(v_{i}\right)-(0)$, and extend this to a homomorphism from $L \uparrow\left\{v_{0}, \ldots, v_{i_{1}}\right\}$ to $L_{c, n+1}$. Now, assume that $\psi$ has been defined on $\left\{v_{i}: i \leq i_{j}\right\}$ for odd $j<l-1$, with $\pi_{c, n, n+1} \circ \psi \uparrow\left\{v_{i}: i \leq i_{j}\right\} \cap B=\phi \uparrow\left\{v_{i}: i \leq i_{j}\right\} \cap B$ remaining true on these vertices. We will extend $\psi$ to $\left\{v_{i}: i \leq i_{j+2}\right\}$. Since $\pi_{c, n, n+1}\left(\psi\left(v_{i_{j}}\right)\right)=\phi\left(v_{i_{j}}\right)$ holds, $\psi\left(v_{i_{j}}\right)$ is of the form $\phi\left(v_{i_{j}}\right)^{-}(\varepsilon)$ for some $\varepsilon \in\{0,1\}$. Note that since $\operatorname{mgs}(B)>2 \cdot \operatorname{length}\left(L_{c, n+1}\right)$, we have that $i_{j+1}-i_{j}>2 \cdot \operatorname{length}\left(L_{c, n+1}\right)$. 
If the parity of the distance between $\phi\left(v_{i_{j}}\right)$ and $\phi\left(v_{i_{j+1}}\right)$ in $L_{c, n}$ is the same as the parity of $i_{j+1}-i_{j}$, then since $i_{j+1}-i_{j}>2 \cdot$ length $\left(L_{c, n+1}\right)>$ length $\left(L_{c, n}\right)$, the map $\psi$ extends to a homomorphism from $L \uparrow\left\{v_{0}, \ldots, v_{i_{j+1}}\right\}$ to $L_{c, n+1}$ with $\psi\left(v_{i_{j+1}}\right)=\phi\left(v_{i_{j+1}}\right)^{\frown}(\varepsilon)$. In this case, define $\psi\left(v_{i}\right)=\phi\left(v_{i}\right)^{\frown}(\varepsilon)$ if $i \in\left[i_{j+1}, i_{j+2}\right]$.

Otherwise, if the parity is different, using $i_{j+1}-i_{j}>2 \cdot \operatorname{length}\left(L_{c, n+1}\right)$ again and the fact that the distance of $\phi\left(v_{i_{j+1}}\right)^{-}(0)$ and $\phi\left(v_{i_{j+1}}\right)^{-}$(1) is odd in $L_{c, n}$ (see (3) of Claim 2.2), we have that $\psi$ can be extended to a homomorphism from $L \uparrow\left\{v_{0}, \ldots, v_{i_{j+1}}\right\}$ to $L_{c, n+1}$ with $\psi\left(v_{i_{j+1}}\right)=\phi\left(v_{i_{j+1}}\right)^{\frown}(1-\varepsilon)$. In this case, define $\psi\left(v_{i}\right)=\phi\left(v_{i}\right)^{\frown}(1-\varepsilon)$ if $i \in\left[i_{j+1}, i_{j+2}\right]$.

This inductive process yields a homomorphism from $L \uparrow\left\{v_{0}, \ldots, v_{i_{l}}\right\}$ to $L_{c, n+1}$ with $\pi_{c, n, n+1} \circ \psi \uparrow$ $\left\{v_{0}, \ldots, v_{m}\right\}=\phi \uparrow\left\{v_{0}, \ldots, v_{i_{l}}\right\}$, and this, of course, can be extended to a homomorphism to the set $\left\{v_{i_{l}}, \ldots, v_{m}\right\}$ (which is disjoint from $B$ ). This finishes the proof of Claim 4.4.

For a given $m$, there are only finitely many homomorphisms from the walk of length $m$ to $L_{c, n+1}$. Fix an enumeration of those homomorphisms $\left(\psi_{j}^{m}\right)_{j<l_{m}}$ for each $m \in \mathbb{N}$. Now, for $x \in B^{\prime}$, let $\left(v_{i}^{x}\right)_{i \leq m^{x}}$ be the enumeration described in (b), and let $j^{x}$ be the minimal index for which $\psi_{j^{x}}^{m^{x}}$ satisfies Claim 4.4. It is clear that the map $x \mapsto \psi_{j^{x}}^{m^{x}}$ is Borel, as is the map $\phi^{\prime}(x)=\psi_{j^{x}}^{m^{x}}(x)$. Moreover, $\phi^{\prime}$ depends only on (a) and (b) and the requirements of the lemma. This finishes the proof of (1).

Now assume that the assumptions of (2) hold, and let $<$ be the lexicographic ordering of $\mathbb{L}_{c}$. It is enough to check that the map $\phi^{\prime}$, defined as in the first part, is a continuous mapping. For a given $x$, $\phi^{\prime}(x)$ depends only on finitely many values. Hence, it suffices to show that if $x_{n} \rightarrow x$, then the values determining $\phi^{\prime}\left(x_{n}\right)$ converge to the values determining $\phi^{\prime}(x)$.

From the definition of $\mathbb{L}_{c^{0}}$, it follows that a connected component of $\mathbb{L}_{c^{0}} \uparrow B^{\prime}$ contains the points of the form $\left\{\left(l, m, t^{\frown} r\right): l<k^{\prime}, m \leq c^{0}(l), t \in 2^{k^{\prime}-l-1}\right\}$ for some $r \in 2^{\mathbb{N}}$. Moreover, if $\left(l_{i}^{n}, m_{i}^{n}, t_{i}^{n}-r^{n}\right) \rightarrow$ $\left(l_{i}, m_{i}, t_{i} \frown r\right)$ for $i<2$ with $l_{i}<k^{\prime}$, then $\left(l_{i}, m_{i}, t_{i} \frown r\right)_{i<2} \in \mathbb{L}_{c}$ iff $\left(\pi_{c^{0}, k^{\prime}-1}\left(l_{i}, m_{i}, t_{i}{ }^{\curlyvee} r\right)\right)_{i<2} \in L_{c^{0}, k^{\prime}-1}$ iff $\left(\pi_{c^{0}, k^{\prime}-1}\left(l_{i}^{n}, m_{i}^{n}, t_{i}^{n} \frown r^{n}\right)\right)_{i<2} \in L_{c^{0}, k^{\prime}-1}$ for every large enough $n$. This and the fact that $<$ is open imply that if $\left(v_{i}^{x_{n}}\right)_{i \leq m^{x_{n}}}$ and $\left(v_{i}^{x}\right)_{i \leq m^{x}}$ are the enumerations of $\mathbb{L}_{c^{0}} \uparrow B^{\prime}$-components described in (b), then $m^{x_{n}}$ must stabilize to $m_{x}$ and $v_{i}^{x_{n}} \rightarrow v_{i}^{x}$ for all $i \leq m_{x}$. Since $B$ is clopen and $\phi$ is continuous, we get that $\phi^{\prime}\left(x_{n}\right)=\phi^{\prime}(x)$ for every large enough $n$.

Proof of Proposition 4.2. We define a sequence $\left(k_{n}, \phi_{n}\right)_{n \in \mathbb{N}}$ inductively. For convenience, we will assume that $B_{0}=\emptyset$. Choose $k_{0}=0$. Then $B_{k_{0}}=\emptyset, \phi_{0}=\emptyset$ and $\operatorname{mgs}\left(B_{k_{0}}\right)=\boldsymbol{\aleph}_{0}>2 \cdot \operatorname{length}\left(L_{c, 1}\right)$. Now assume that $\left(\phi_{i}, k_{i}\right)_{i \leq n}$ has already been defined with the properties that $\operatorname{mgs}\left(B_{k_{n}}\right)>2 \cdot \operatorname{length}\left(L_{c, n+1}\right)$ and $\phi_{i}$ is a Borel homomorphism from $L \uparrow B_{k_{i}}$ to $L_{c, i}$. Choose $k_{n+1}$ so large that $\operatorname{mgs}\left(B_{k_{n+1}}\right)>$ $2 \cdot$ length $\left(L_{c, n+2}\right)$. An application of (1) of Lemma 3.6 to $B_{k_{n}}, B_{k_{n+1}}, n$ and $\phi_{n}$ yields a homomorphism $\phi_{n+1}$ of $L \uparrow B_{k_{n+1}}$ to $L_{c, n+1}$ so that $\pi_{c, n, n+1} \circ \phi_{n+1} \uparrow B_{k_{n}}=\phi_{n}$. Thus, we obtain a sequence $\left(k_{n}, \phi_{n}\right)_{n \in \mathbb{N}}$ that satisfies the assumptions (1)-(3) of Claim 2.4, which finishes the proof of the first part.

Finally, a similar proof yields the second half: first, note that if $B=\left\{(l, m, r) \in \mathbb{X}_{c^{0}}: l<k\right\}$, then $\operatorname{mgs}(B)=\min \{c(i)+1: i \geq k\}$. This and the assumption that $c^{0}(n) \rightarrow \infty$ allow us to find the sequence $\left(B_{k_{n}}\right)_{n \in \mathbb{N}}$ and iterate (2) of Lemma 4.3. This yields a sequence $\left(k_{n}, \phi_{n}\right)_{n \in \mathbb{N}}$ satisfying (1)-(4) of Claim 2.4.

Combining the preceding theorems, we obtain the following result, which, of course, implies Theorem 1.1.

Theorem 4.5. Assume that $G$ is an analytic graph on a Hausdorff space. Then exactly one of the following holds.

(1) $\chi_{B}(G) \leq 2$ : that is, $G$ is Borel bipartite.

(2) $\mathbb{L}_{0}$ admits a continuous homomorphism to $G$. 
Proof. The fact that (1) and (2) are mutually exclusive follows from the observations that $\chi_{B}\left(\mathbb{L}_{0}\right)>2$ ((7) of Claim 2.2) and $\mathbb{L}_{0} \leq_{c} G \Longrightarrow \chi_{B}\left(\mathbb{L}_{0}\right) \leq \chi_{B}(G)$.

Now assume that (1) is false. Recall that $\mathbb{L}_{0}=\mathbb{L}_{c^{0}}$, where $c^{0}(0)=1$ and $c^{0}(n)=2 n-1$. Then, by Theorem 3.1, there exists an odd $\boldsymbol{\aleph}_{0}$-pair $b=(c, d)$ such that $\mathbb{L}_{b} \leq_{c} G$. But then $\mathbb{L}_{c} \leq_{c} G$, and using (2) of Proposition 4.2, we obtain $\mathbb{L}_{0} \leq_{c} \mathbb{L}_{c} \leq_{c} G$.

We conclude this section by proving:

Theorem 1.2. Suppose that $X$ is a standard Borel space and $L$ is an acyclic Borel graph on $X$ of vertex degree at most two. Then the following are equivalent:

(1) There is a Borel homomorphism from L to every Borel graph $G$ of Borel chromatic number at least three.

(2) The graph L has the large gap property modulo a two-colorable set.

Proof. First, assume (2). Using Claim 4.1 together with (1) of 4.2 , we obtain a sequence $\left(B_{n}\right)_{n \in \mathbb{N}}$ of Borel sets and an $L$-invariant Borel set $M$ such that $L \uparrow \cup_{n} B_{n} \leq_{B} \mathbb{L}_{0}, E_{L} \uparrow X \backslash\left(M \cup \cup_{n} B_{n}\right)$ is smooth, $X \backslash \cup_{n} B_{n}$ is $L$-invariant and $\chi_{B}(L \uparrow M) \leq 2$. By Claim 2.3 and the invariance of $M$, we have that $\chi_{B}\left(L \uparrow X \backslash \bigcup_{n} B_{n}\right) \leq 2$, so $L \uparrow X \backslash \bigcup_{n} B_{n}$ admits a Borel homomorphism to each non-empty Borel graph. Putting together the Borel homomorphisms on the invariant sets $X \backslash \bigcup_{n} B_{n}$ and $\bigcup_{n} B_{n}$, we obtain $L \leq_{B} \mathbb{L}_{0}$. Thus, by Theorem 4.5, $L$ admits a Borel homomorphism to each Borel graph with Borel chromatic number $>2$.

Now assume that $L \leq_{B} \mathbb{L}_{0}$, as witnessed by the Borel map $\phi$. Let $M$ be the set from (2) of Claim 2.3, and let $B \subseteq \mathbb{X}_{0}$ witness that $\mathbb{L}_{0}$ has the large gap property ((8) of Claim 2.2). To show the theorem, it is enough to check that $L \uparrow X \backslash M$ has the large gap property. Letting $B^{\prime}=(\phi \uparrow X \backslash M)^{-1}(B)$, it is easy to see from the fact that $\phi \uparrow X \backslash M$ maps $L$-components onto $\mathbb{L}_{0}$-components that $B^{\prime}$ witnesses the large gap property for $L \uparrow X \backslash M$.

\section{An antibasis result for digraphs}

Finally, we show a slightly more general version of Theorem 1.3:

Theorem 5.1. Suppose that $G$ is an analytic digraph on a Hausdorff space with $\chi_{B}(G)>2$. Then there is a sequence $\left(L_{t}\right)_{t \in 2^{\mathbb{N}}}$ of Borel oriented graphs on Polish spaces such that for each $t \in 2^{\mathbb{N}}$, we have $L_{t} \leq_{c} G$ and $\chi_{B}\left(L_{t}\right)>2$, and any analytic digraph on a Hausdorff space that admits a Borel homomorphism to at least two oriented graphs of the form $L_{t}$ has a Borel two-coloring.

Let us start with some definitions. Assume that $G$ is an acyclic oriented graph on a space $X$, and let $B \subseteq X$. Using Claim 2.1, we can define the didistance set of $B$ by letting $D^{G}(B)=\{n \in \mathbb{Z}: \exists x, y \in$ $B$ didist $\left.^{G}(x, y)=n\right\}$.

Lemma 5.2. Assume that $L$ is $a \leq 2$-regular acyclic Borel oriented graph on the space $X, b=(c, d)$ is an odd $\boldsymbol{\aleph}_{0}$-pair and $\mathbb{L}_{b} \leq_{B}$ L. Assume that $C$ is a Borel $E_{L}$-complete set. There exists a non-meager Borel set $B \subseteq \mathbb{X}_{c}$ such that $D^{\mathbb{L}_{b}}(B) \subseteq D^{L}(C)$.

Proof. Let $\phi$ be a Borel homomorphism from $\mathbb{L}_{b}$ to $L$, and let $M=\left\{x \in \mathbb{X}_{c}: \phi\right.$ mapping $[x]_{E} \rightarrow$ $[\phi(x)]_{E_{L}}$ is not onto\}. By Claim 2.3, we have $\chi_{B}\left(\mathbb{L}_{b} \uparrow M\right) \leq 2$. By the invariance of $M$ and (7) of Claim 2.2, it must be meager. Define $B=\phi^{-1}(C) \backslash M$; we will check that $B$ is non-meager. Note that, as $C$ is an $E_{L}$-complete set, the set $B$ is a $E_{c} \uparrow\left(\mathbb{X}_{c} \backslash M\right)$-complete. As $[B]_{E_{c}} \supseteq \mathbb{X}_{c} \backslash M$ is comeager, it follows from (6) of Claim 2.2 that $B$ cannot be meager.

Finally, if $x, y \in B$, let $p=\left(\left(z_{0}, \ldots, z_{l}\right), d_{p}\right)$ be an $\mathbb{L}_{b}$-walk with $z_{0}=x$ and $z_{l}=y$. Then $p^{\prime}=\left(\left(\phi\left(z_{0}\right), \ldots, \phi\left(z_{l}\right)\right), d_{p}\right)$ is an $L$-walk with $\operatorname{dilength}\left(p^{\prime}\right)=\operatorname{dilength}(p)$. It follows that $\operatorname{didist}^{\mathbb{L}_{b}}(\phi(x), \phi(y)) \in D^{L}(C)$. Thus $D^{\mathbb{L}_{b}}(B) \subseteq D^{L}(C)$. 
To carry out our construction, we will impose a growth condition on the approximations to our graphs. Assume that $b=(c, d)$ is an odd $\boldsymbol{\aleph}_{0}$-pair. We say that $b$ has property $(*)$ if for every $i \in \mathbb{N}$, we have

$$
\Sigma(d(i))>8 \cdot \sum_{j<i} 2^{i-j} \cdot|\Sigma(d(j))| .
$$

Lemma 5.3. Suppose that $b=(c, d)$ is an odd $\boldsymbol{\aleph}_{0}$-pair with property $(*)$. Then there exists a collection $\left(P_{t}\right)_{t \in 2^{\mathbb{N}}}$ of perfect subsets of $\mathbb{X}_{c}$ such that, for every $t \neq t^{\prime}$, we have

(1) $\left|D^{\mathbb{L}_{b}}\left(P_{t}\right)\right|=\boldsymbol{\aleph}_{0}$.

(2) There exists an $i_{0} \in \mathbb{N}$ such that, for every $k, k^{\prime} \geq i_{0}$, if $k \in D^{\mathbb{L}_{b}}\left(P_{t}\right)$ and $k^{\prime} \in D^{\mathbb{L}_{b}}\left(P_{t^{\prime}}\right)$, then $\frac{k}{k^{\prime}} \notin\left[\frac{1}{4}, 4\right]$.

(3) $\chi_{B}\left(\mathbb{L}_{b} \uparrow\left[P_{t}\right]_{E_{c}}\right)=3$.

Proof. Let $S \subseteq 2^{\mathbb{N}}$ be a perfect almost disjoint family of infinite sets (identifying $2^{\mathbb{N}}$ with $\mathcal{P}(\mathbb{N})$ ). Of course (using a bijection between $S$ and $2^{\mathbb{N}}$ ), it is enough to construct a family indexed by the elements of $S$.

For $t \in S$, let

$$
P_{t}=\left\{(n, k, r) \in \mathbb{X}_{c}: n=k=0, \forall i \in \mathbb{N}(t(i)=0 \Longrightarrow r(i)=0)\right\} .
$$

Claim. Assume that $x \neq y \in P_{t}$ and $x E_{c} y$. Let $x=\left(0,0, r^{x}\right)$ and $y=\left(0,0, r^{y}\right)$, and let $i \in \mathbb{N}$ be maximal with $r^{x}(i) \neq r^{y}(i)$ (such an $i$ exists since $x E_{c} y$ ). Then $t(i)=1$ and $\left|\operatorname{didist}^{\mathbb{L}_{b}}(x, y)\right| \in$ $\left[\frac{\Sigma(d(i))}{2}, 2 \cdot \Sigma(d(i))\right]$.

Proof. It is obvious from the definition of $P_{t}$ that $t(i)=1$.

Moreover, it follows from the definition of $\mathbb{L}_{b}$ and the choice of $i$ that $\operatorname{didist}^{\mathbb{L}_{b}}(x, y)=$ $\operatorname{didist}^{L_{b, i}}\left(\pi_{c, i}(x), \pi_{c, i}(y)\right)$, so it is enough to give an estimate of the latter. Since $r^{x}(i) \neq r^{y}(i), \pi_{c, i}(x)$ and $\pi_{c, i}(y)$ are in different copies of $L_{b, i-1}$ in $L_{b, i}$. But then

$$
\left.\Sigma(d(i))-\left|\operatorname{dilength}\left(L_{b, i-1}\right)\right|\right) \leq\left|\operatorname{didist}\left(\pi_{c, i}(x), \pi_{c, i}(y)\right)\right| \leq \Sigma(d(i))+\left|\operatorname{dilength}\left(L_{b, i-1}\right)\right| .
$$

So, by an easy induction, we have

$$
\Sigma(d(i))-\sum_{j<i} 2^{i-j} \cdot \Sigma(d(j)) \leq\left|\operatorname{didist}\left(\pi_{c, i}(x), \pi_{c, i}(y)\right)\right| \leq \Sigma(d(i))+\sum_{j<i} 2^{i-j} \cdot \Sigma(d(j)),
$$

which implies our statement, by $(*)$.

The claim clearly implies property (1).

Assume that $t \neq t^{\prime}$ are given. By the choice of $S$, there exists an $i_{0}^{*} \in \mathbb{N}$ such that $t \cap t^{\prime} \subseteq i_{0}^{*}$. Let $i_{0}=\frac{\Sigma\left(d\left(i_{0}^{*}\right)\right)}{2}$, and assume that $k, k^{\prime} \geq i_{0}$, with $k \in D^{\mathbb{L}_{b}}\left(P_{t}\right), k^{\prime} \in D^{\mathbb{L}_{b}}\left(P_{t^{\prime}}\right)$. The choice of $i_{0}^{*}$ and $i_{0}$, together with the claim and $(*)$, yields that $k \in\left[\frac{\Sigma(d(i))}{2}, 2 \cdot \Sigma(d(i))\right]$ and $k^{\prime} \in\left[\frac{\Sigma\left(d\left(i^{\prime}\right)\right)}{2}, 2 \cdot \Sigma\left(d\left(i^{\prime}\right)\right)\right]$ with $i \neq i^{\prime}$. But then $\frac{k}{k^{\prime}} \notin\left[\frac{1}{4}, 4\right]$ follows from $(*)$, showing (2).

Finally, a Baire category argument analogous to the one in the proof of (7) of Claim 2.2 yields that (3) holds for each $t \in S$.

Lemma 5.4. Assume that $b^{*}=\left(c^{*}, d^{*}\right)$ is an odd $\boldsymbol{\aleph}_{0}$-pair with property $(*)$, and $B \subseteq \mathbb{X}_{c^{*}}$ is a Borel set so that the set $[B]_{E_{c^{*}}}$ is comeager. Then there exists an $i_{1} \in \mathbb{N}$ such that, for every $i>i_{1}$, we have that

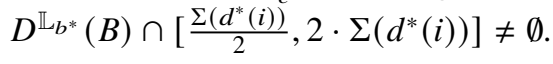

Proof. By our assumption on $B$ and (6) of Claim 2.2, we can find an $i_{1}$ and a non-empty basic open set of the form $\left[\left(i_{1}, k, \sigma\right)\right]$ in which $B$ is comeager. Let $i>i_{1}$. By shrinking $B$ with the $E_{c^{*}}$-saturation of the meager set $[(i, k, \sigma)] \backslash B$ (which is also a meager set), we can assume that $[(i-1, k, \sigma)] \cap B=[(i-$ 
$1, k, \sigma)] \cap[B]_{E_{c^{*}}}$ In particular, we can find an $r \in 2^{\mathbb{N}}$ so that $\left(i-1, k, \sigma^{\frown}(0)^{\frown} r\right),\left(i-1, k, \sigma^{\frown}(1)^{\complement} r\right) \in B$. Let $x=\left(i-1, k, \sigma^{\frown}(0)^{\complement} r\right)$ and $y=\left(i-1, k, \sigma^{\frown}(1)^{\frown} r\right)$.

Again, it is clear that didist $\mathbb{L}_{b^{*}}(x, y)=\operatorname{didist}^{L_{b^{*}, i}}\left(\pi_{c, i}(x), \pi_{c, i}(y)\right)$. Moreover, $\pi_{c, i}(x)$ and $\pi_{c, i}(y)$ are in different copies of $L_{b^{*}, i-1}$ in $L_{b^{*}, i}$. So

$$
\Sigma\left(d^{*}(i)\right)-\sum_{j<i} 2^{i-j} \cdot \Sigma\left(d^{*}(j)\right) \leq\left|\operatorname{didist}\left(\pi_{c, i}(x), \pi_{c, i}(y)\right)\right| \leq \Sigma\left(d^{*}(i)\right)+\sum_{j<i} 2^{i-j} \cdot \Sigma\left(d^{*}(j)\right),
$$

which implies our statement, by $(*)$.

Proof of Theorem 5.1. By Theorem 3.1, without loss of generality, we can assume that $G=\mathbb{L}_{b}$ for some odd $\boldsymbol{\aleph}_{0}$-pair $b=(c, d)$ with property $(*)$. Now, using Lemma 5.3 , we obtain a family $\left(P_{t}\right)_{t \in 2^{\mathbb{N}}}$ of perfect subsets of $\mathbb{X}_{c}$ having properties (1)-(3). For each $t \in 2^{\mathbb{N}}$, let $L_{t}=\mathbb{L}_{b} \uparrow\left[P_{t}\right]_{E_{c}}$. We show that $\left(L_{t}\right)_{t \in 2^{\mathbb{N}}}$ satisfies the requirements of the theorem. The condition on the Borel chromatic numbers is clear from (3) of Lemma 5.3.

Let $t, t^{\prime} \in 2^{\mathbb{N}}$ be distinct. Assume that $H \leq_{B} L_{t}, L_{t^{\prime}}$ with $\chi_{B}(H)=3$. Then, by Theorem 3.1, we can assume that $H=\mathbb{L}_{b^{*}}$ and $b^{*}$ has property $(*)$. As $P_{t}$ and $P_{t^{\prime}}$ are $E_{L_{t}}$ - and $E_{L_{t^{\prime}}}$-complete sets, using Lemma 5.2, we obtain non-meager Borel sets $B, B^{\prime}$ in $\mathbb{X}_{c^{*}}$ with $D^{\mathbb{L}_{b^{*}}}(B) \subseteq D^{L_{t}}\left(P_{t}\right)$ and $D^{\mathbb{L}_{b^{*}}}\left(B^{\prime}\right) \subseteq D^{L_{t^{\prime}}}\left(P_{t^{\prime}}\right)$. Let $i>i_{0}, i_{1}, i_{1}^{\prime}$, where $i_{0}$ comes from (2) of Lemma 5.3, while $i_{1}, i_{1}^{\prime}$ are obtained from applying Lemma 5.4 to $B$ and $B^{\prime}$.

By Lemma 5.4, we can find $\left.k \in D^{\mathbb{L}_{b^{*}}}(B) \cap\left[\frac{\Sigma\left(d^{*}(i)\right)}{2}, 2 \cdot \Sigma\left(d^{*}(i)\right)\right)\right], k^{\prime} \in D^{\mathbb{L}_{b^{*}}}\left(B^{\prime}\right) \cap\left[\frac{\Sigma\left(d^{*}(i)\right)}{2}, 2\right.$. $\left.\Sigma\left(d^{*}(i)\right)\right]$. But then $\frac{k}{k^{\prime}} \in\left[\frac{1}{4}, 4\right]$, which contradicts $k \in D^{L_{t}}\left(P_{t}\right), k^{\prime} \in D^{L_{t^{\prime}}}\left(P_{t^{\prime}}\right)$ and (2) of Lemma 5.3.

\section{Open problems}

We conclude with a number of open problems. First, it is not clear how Theorem 1.2 can be generalized to arbitrary Borel graphs.

Problem 6.1. Characterize the Borel graphs with Borel chromatic number 3 that admit a Borel homomorphism to each Borel graph $G$ with $\chi_{B}(G)>2$ (or, equivalently, those that are $\leq_{B} \mathbb{L}_{0}$ ).

The product of graphs $G$ on $X$ and $G^{\prime}$ on $X^{\prime}$ is the graph on $X \times X^{\prime}$ given by $\left(\left(x, x^{\prime}\right),\left(y, y^{\prime}\right)\right) \in$ $G \times G^{\prime} \Longleftrightarrow(x, y) \in G$ and $\left(x^{\prime}, y^{\prime}\right) \in G^{\prime}$. The Borel version of Hedetniemi's conjecture reads as follows: is $\chi_{B}\left(G \times G^{\prime}\right)=\min \left\{\chi_{B}(G), \chi_{B}\left(G^{\prime}\right)\right\}$ ?

Theorem 1.1 implies that the answer is affirmative when $\min \left\{\chi_{B}(G), \chi_{B}\left(G^{\prime}\right)\right\} \leq 3$. El-Zahar and Sauer [9] showed that, for finite graphs, the bound 4 already implies an affirmative answer. Hence the following problem is quite natural.

Problem 6.2. Assume that $G, G^{\prime}$ are Borel graphs on standard Borel spaces and $\min \left\{\chi_{B}(G), \chi_{B}\left(G^{\prime}\right)\right\} \leq 4$. Is $\chi_{B}\left(G \times G^{\prime}\right)=\min \left\{\chi_{B}(G), \chi_{B}\left(G^{\prime}\right)\right\}$ ?

A recent breakthrough result of Shitov [24] ensures that the answer is negative in general; there is a counterexample for finite graphs.

The $\mathbb{G}_{0}$ dichotomy, the results in [25] and the current paper give a complete description of the existence of simple bases for Borel graphs with a given Borel chromatic number. However, the natural reformulation of the notion of chromatic numbers in terms of homomorphisms raises the following problem:

Problem 6.3. Characterize the Borel graphs $H$ so that the collection $\left\{G: G\right.$ is a Borel graph, $\left.G \bigsqcup_{B} H\right\}$ has a single element basis.

It is conceivable that such a characterization is impossible due to a complexity barrier. 
Babai's celebrated results [1] suggest that, among finite graphs, the isomorphism relation is simpler than the homomorphism relation. It would be interesting to know the answer to the analogous question for Borel graphs.

Problem 6.4. Determine the projective complexity of the isomorphism relation for Borel graphs on Polish spaces.

Acknowledgements. The authors were supported in part by FWF Grants P28153 and P29999. R. Carroy was supported by PRIN grant number 2017NWTM8R. Z. Vidnyánszky was also supported by NKFIH, grant nos. 113047 and 129211 and FWF Grant M2779.

Conflict of Interest: None.

\section{References}

[1] L. Babai. Graph isomorphism in quasipolynomial time. In Proceedings of the forty-eighth annual ACM symposium on Theory of Computing, pages 684-697. ACM, 2016.

[2] A. Bernshteyn. Measurable versions of the Lovász local lemma and measurable graph colorings. arXiv preprint arXiv:1604.07349, 2016.

[3] R. Carroy, B.D. Miller, D. Schrittesser and Z. Vidnyanszky. Minimal definable graphs with no definable two-colorings. 2018. http://www.logic.univie.ac.at/ vidnyanszz77/summary2.pdf.

[4] J.D. Clemens, C.T. Conley and B.D. Miller. The smooth ideal. Proc. Lond. Math. Soc. (3), 112(1): 57-80, 2016.

[5] J.D. Clemens, D. Lecomte and B.D. Miller. Essential countability of treeable equivalence relations. Adv. Math., 265: 1-31, 2014.

[6] C.T. Conley and A.S. Kechris. Measurable chromatic and independence numbers for ergodic graphs and group actions. Groups Geom. Dyn., 7(1): 127-180, 2013.

[7] C.T. Conley and B.D. Miller. An antibasis result for graphs of infinite Borel chromatic number. Proc. Amer. Math. Soc., 142(6): 2123-2133, 2014.

[8] E. Csóka, Ł. Grabowski, A. Máthé, O. Pikhurko and K. Tyros. Borel version of the local lemma. arXiv preprint arXiv:1605.04877, 2016.

[9] M. El-Zahar and N.W. Sauer. The chromatic number of the product of two 4-chromatic graphs is 4. Combinatorica, 5(2): 121-126, 1985.

[10] S. Gao. Invariant descriptive set theory, volume 293 of Pure and Applied Mathematics (Boca Raton). CRC Press, Boca Raton, FL, 2009.

[11] L.A. Harrington, A.S. Kechris and A. Louveau. A Glimm-Effros dichotomy for Borel equivalence relations. J. Amer. Math. Soc., 3(4): 903-928, 1990.

[12] A S. Kechris. Classical descriptive set theory, volume 156 of Graduate Texts in Mathematics. Springer-Verlag, New York, 1995.

[13] A.S. Kechris and A.S. Marks. Descriptive graph combinatorics. 2015. http://math.ucla.edu/ marks/.

[14] A.S. Kechris, S. Solecki and S. Todorcevic. Borel chromatic numbers. Adv. Math., 141(1): 1-44, 1999.

[15] D. Lecomte and M. Zeleny. Baire-class $\xi$ colorings: the first three levels. Trans. Amer. Math. Soc., 366(5): $2345-2373,2014$.

[16] D. Lecomte and M. Zeleny. Analytic digraphs of uncountable Borel chromatic number under injective definable homomorphism. arXiv preprint arXiv:1811.04738, 2018.

[17] A.S. Marks. A determinacy approach to Borel combinatorics. J. Amer. Math. Soc., 29(2): 579-600, 2016.

[18] A.S. Marks and S.T. Unger. Borel circle squaring. Ann. of Math. (2), 186(2): 581-605, 2017.

[19] B.D. Miller. The graph-theoretic approach to descriptive set theory. Bull. Symbolic Logic, 18(4): 554-575, 2012.

[20] B.D. Miller. An introduction to classical descriptive set theory. Lecture notes, 2015.

[21] B.D. Miller. Lacunary sets and actions of tsi groups. Preprint, 2018.

[22] B.D. Miller and Z. Vidnyánszky. On the existence of large antichains for definable quasi-orders. J. Symb. Log., 85(1): 103-108, 2020.

[23] Y. Pequignot. Finite versus infinite: an insufficient shift. Adv. Math., 320(1): 244-249, 2017.

[24] Y. Shitov. Counterexamples to Hedetniemi's conjecture. arXiv preprint arXiv:1905.02167, 2019.

[25] S. Todorčević and Z. Vidnyanszky. A complexity problem for Borel graphs. submitted, 2017. 